\title{
Long term efficacy of anti-PD1 therapy in Hodgkin Lymphoma with and without allogenic stem cell transplantation
}

Guillaume Manson ${ }^{1}$, Jean-Baptiste Mear ${ }^{1}$, Charles Herbaux ${ }^{2}$, Jean-Marc Schiano ${ }^{3}$, Olivier Casasnovas $^{4}$, Aspasia Stamatoullas ${ }^{5}$, Bénédicte Deau ${ }^{6}$, Anna Schmitt ${ }^{7}$, Georges Garnier ${ }^{8}$, Caroline Regny ${ }^{9}, K^{3}$ rimo Bouabdallah ${ }^{10}$, Marie-Pierre Moles-Moreau ${ }^{11}$, Hervé Ghesquieres ${ }^{12}$, Adrian Tempescul ${ }^{13}$, Remy Dulery ${ }^{14}$, Emmanuelle Nicolas-Virelizier ${ }^{15}$, Alain Delmer ${ }^{16}$, Cecile Borel $^{17}$, Adrien Chauchet ${ }^{18}$, Diane Damotte ${ }^{19}$, Laurent Dercle ${ }^{20-22}$, Pauline Brice ${ }^{23}$ and Roch Houot $^{1,24}$, on behalf of the LYSA

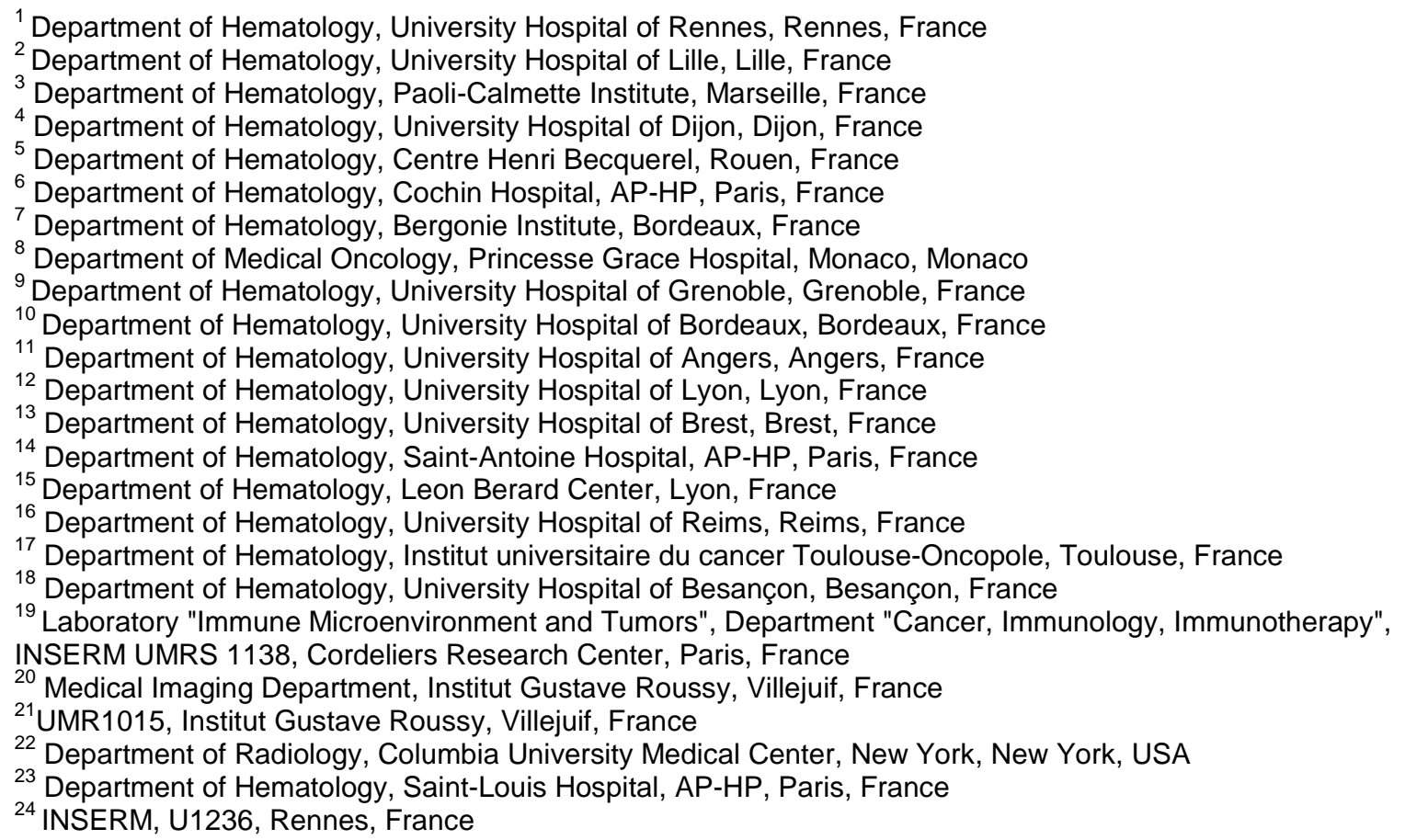

\section{Corresponding author:}

Prof. Roch Houot

Department of Hematology

CHU Rennes

2 rue Henri Le Guilloux

35033 Rennes Cedex 9

France

Tel: +33 (0)2 99284226

Fax: +33 (0)2 99284161

E-mail: roch.houot@chu-rennes.fr 
Running title: Anti-PD1 and transplantation in Hodgkin lymphoma

\title{
Highlights:
}

- The majority of R/R HL patients eventually progressed during anti-PD1 therapy.

- In responding patients, the relapse rate was lower in patients consolidated with alloHSCT.

- Among responders, subsequent allo-HSCT was not associated with a greater overall survival.

Keywords: Immunotherapy; Hodgkin Lymphoma; Checkpoint inhibitors; anti-PD1; nivolumab; Allogenic hematopoietic stem cell transplantation.

\author{
Abstract word count: 252 words \\ Text word count: 2750 words \\ Figures: 3 \\ Tables: 2 \\ Supplementary Tables \& Figures: 6 \\ References: 24
}




\section{Abstract}

Introduction: Long term efficacy of anti-PD1 therapy and the need for a consolidation with allogenic stem cell transplantation (alloHSCT) remain unclear in patients with relapsed/refractory $(\mathrm{R} / \mathrm{R})$ Hodgkin lymphoma $(\mathrm{HL})$.

Methods: We retrospectively analyzed 78 patients with $\mathrm{R} / \mathrm{R} \mathrm{HL}$ treated with nivolumab in the French Early Access Program and compared their outcome according to subsequent alloHSCT.

Results: After a median follow-up of 34.3 months, the best overall response rate was $65.8 \%$, including $38.2 \%$ complete responses (CR). The median progression-free survival (PFS) was 12.1 months. Patients reaching a CR upon nivolumab had a significantly longer PFS than those reaching a partial response $(P R)$ (median = not reached vs 9.3 months, $p<0.001)$. In our cohort, 13 patients who responded (i.e. in $\mathrm{CR}$ or $\mathrm{PR}$ ) to nivolumab monotherapy underwent consolidation with alloHSCT. Among responding patients, none of those who underwent subsequent alloHSCT $(\mathrm{N}=13)$ relapsed, whereas $62.2 \%$ of those who were not consolidated with alloHSCT $(\mathrm{N}=37)$ relapsed $(\mathrm{p}<0.001)$. There was no difference in overall survival (OS) between the two groups. Five out of 6 patients who were not in $\mathrm{CR}$ at the time of transplantation (4 PR and $1 \mathrm{PD}$ ) converted into a CR after alloHSCT.

Conclusion: Most R/R HL patients treated with anti-PD1 monotherapy eventually progressed, notably those who did not achieve a CR. Patients undergoing consolidation with alloHSCT after anti-PD1 therapy experienced prolonged disease-free survival compared to non-transplanted patients, but this difference did not translate into a benefit in OS. This information should be considered when evaluating the risk/benefit ratio of alloHSCT after anti-PD1 therapy. 
2 Classical Hodgkin Lymphoma $(\mathrm{HL})$ is highly curable with standard treatments. However, 3

\section{Introduction}

relapse can occur in up to $30 \%$ of patients with advanced disease[1,2]. In relapsed patients, salvage therapy followed by autologous hematopoietic stem cell transplantation (autoHSCT) can induce prolonged remissions[3]. Patients who relapse after autoHSCT have a poor outcome with a median overall survival (OS) ranging from 10.5 to 27.6 months in the prebrentuximab-vedotin era[4,5]. Although brentuximab-vedotin has shown encouraging overall response rates in relapsed or refractory $(R / R) H L$, only $~ 20 \%$ of patients might be cured with this single-agent therapy[6].

In recent years, checkpoint blockade with anti-PD1 antibodies demonstrated remarkable efficacy in patients with $\mathrm{R} / \mathrm{R} \mathrm{HL}[7-10]$. Studies evaluating anti-PD1 therapy in these patients, which account for more than 450 patients in total, showed overall response rates (ORR) of around $70 \%$ and complete remissions (CR) of up to $20 \%$. These results led to the approval of nivolumab and pembrolizumab for R/R HL by the Food and Drug Administration in 2016 and 2017, respectively. In 2015, an Early Access Program (EAP) was started in France allowing treatment of $\mathrm{R} / \mathrm{R} \mathrm{HL}$ patients with nivolumab.

Despite remarkable immediate efficacy, many questions remain unanswered regarding the long-term efficacy and optimal management of HL patients treated with anti-PD1, including the duration of treatment and the need for a consolidation with allogenic stem cell transplantation (alloHSCT). We have previously addressed the first issue and demonstrated that prolonged remissions can be achieved after nivolumab discontinuation in patients with CR[11]. Here, we evaluated the role of alloHSCT consolidation in HL patients treated with anti-PD1.

There is limited data regarding the long term efficacy of anti-PD1 therapy since the median follow-up of previously published studies was rather short (median = 7-18 months[10,12,13]). Furthermore, the role of alloHSCT after anti-PD1 therapy remains poorly defined. A 
1 retrospective study by Merryman et al ( $\mathrm{N}=31 \mathrm{HL}$ patients) suggested that patients

2 undergoing alloHSCT after nivolumab might experience a lower relapse rate compared to

3 historical controls[14]. However, this strategy was not compared to patients receiving anti-

$4 \quad$ PD1 treatment without subsequent alloHSCT.

5 Here, we report the results of R/R HL patients consolidated with alloHSCT after nivolumab treatment in comparison to patients treated with nivolumab monotherapy alone in the French EAP.

\section{Methods}

We conducted a retrospective, nationwide study of patients aged $\geq 18$ years with $R / R \mathrm{HL}$ who were treated with nivolumab in the French EAP. EAP included patients with $\mathrm{HL}$ relapsing or refractory after three lines of chemotherapy (including Brentuximab-Vedotin) and autoHSCT, or four lines of chemotherapy if the patient was not eligible for HSCT due to age, insufficient stem-cell collection or chemorefractory disease. Patients with active auto-immune disease and/or steroid or immunosuppressive treatment-requiring disease were not eligible. All patients who had received at least one dose of nivolumab as part of the French EAP were eligible for the study. Nivolumab was administered at $3 \mathrm{mg} / \mathrm{kg}$ IV over 60 min every 2 weeks in an outpatient setting until progression, death of any cause, unacceptable toxicity, consent withdrawal, or treating physician's decision. Patients were allowed to undergo subsequent alloHSCT according to primary physician's decision.

Primary endpoint was best response (ORR, CR, partial response (PR), stable disease (SD) and progressive disease (PD) as defined by the primary physician using the Cheson 1999[15] or Cheson 2007[16] criteria depending on the current practice in each center at the time of evaluation) at any time during nivolumab treatment.

Secondary endpoints included other efficacy parameters (including duration of response, progression-free survival, and overall survival), safety analysis and the impact of alloHSCT. 
1 OS and progression-free survival (PFS) were defined as the duration from the first dose of

2 nivolumab to death of any cause and disease progression or death of any cause, whichever occurred first, respectively. Both OS and PFS were censored at the date of last information and were estimated using the Kaplan-Meier method. Exact 95\% confidence intervals (Cl) were used when appropriate. All data analysis was carried out using SAS v.9.3 software. Safety and tolerability were evaluated and reported by investigators according to the National Cancer Institute Common Terminology Criteria for Adverse Events version 4.03.

The protocol was approved by the Comité Consultatif sur le Traitement de l'Information en matière de Recherche dans le domaine de la Santé - CCTIRS (Approval n 16.861). All patients have been informed and consented before registration.

\section{Results}

\section{Patients' characteristics}

Seventy-eight patients from 35 French centers were included (Supplementary Figure 1). The characteristics of the patients are summarized in Table 1. Median age at nivolumab initiation was 37 (range, 18 - 77) years. Performance status was $\geq 2$ in $27 \%$ of the patients, $27 \%$ of them had B symptoms and $77.5 \%$ had stage III/IV disease. The median number of prior lines of treatment was 6 (range, $2-13$ ). All patients had been previously treated with brentuximab-vedotin, $62 \%$ and $28 \%$ of them had undergone prior autologous or allogenic HSCT, respectively. The median exposure to nivolumab was 5.2 (range, $0-38.3$ ) months, and the median number of cycles was 9.5 (range, $1-84$ ). The median follow-up was 34.3 months (range, $0.1-39.7$ ). At the time of analysis, $85.9 \%$ of patients had discontinued nivolumab, mostly because of progression $(50.7 \%)$, consolidation with alloHSCT $(19.4 \%)$ or toxicity $(9.0 \%)$.

\section{Outcome of the entire cohort}


1 Among evaluable patients $(n=76)$, the best ORR was $65.8 \%(90 \% \mathrm{Cl}[55.82 ; 74.80])$,

2 including $38.2 \% \mathrm{CR}$ and $27.6 \% \mathrm{PR}$. The median time to response was 2.6 months $(95 \% \mathrm{Cl}$

$3[2.0 ; 4.0])$ and the median time to best response was 2.7 months (6 patients reached CR at

4 the second evaluation and 2 others later). The median duration of response was 24.3 months

5 (95\% Cl [9.9; not evaluable]). Median PFS was 12.1 months (95\% Cl [7.3; 26.2]) and median

6 OS was 38.7 months $(95 \% \mathrm{Cl}$ [38.7; not evaluable]). At three years of follow-up, the PFS and

7 OS rates were $32 \%$ and $65 \%$, respectively (Figure 1 A \& B). Patients achieving a CR upon

8 nivolumab had a significantly longer PFS than those reaching a PR (median PFS $=$ not reached vs 9.3 months, $\mathrm{p}<0.001)$. There was no difference in OS between those two groups

(Figure $1 \mathrm{C}$ \& D). Among 50 responders (CR or PR), 23 (46\%) patients had relapsed/progressed at the time of analysis: 7 out of 29 (24\%) CR patients, 16 out of 21 (76\%) PR patients, and up to $94 \%$ of the PR patients who did not undergo subsequent alloHSCT. Overall, 45 (57.7\%) patients relapsed or progressed after nivolumab treatment. Thirty-six $(80 \%)$ of them received a salvage therapy. Among 23 patients evaluated, 13 (56.6\%) experienced an objective response and 8 (34.8\%) achieved CR.

A total of 107 adverse events (AE) were reported in 40 (51.3\%) patients. Among these, there were $62 \mathrm{AE}$ of grade $\geq 3$ in $29(37 \%)$ patients and 28 serious $\mathrm{AE}(\mathrm{SAE})$ in $16(20.5 \%)$ patients. Most frequent clinically relevant, immune-related and serious AE are summarized in Supplementary Table 1.

At the time of analysis, $54(69.2 \%)$ patients were alive. The main cause of death was lymphoma progression in 14 (58.3\%) patients.

\section{Outcome of patients according to subsequent alloHSCT consolidation}

In our cohort, 17 patients proceeded to alloHSCT after nivolumab therapy (Figure $2 \& 3$, Supplementary Table 2). One patient reached a PR upon nivolumab then progressed before starting the conditioning regimen and thus received radiotherapy before transplantation. Three patients had a PD upon nivolumab therapy of whom 2 received a salvage therapy 
1 before transplantation. Characteristics of allo-transplanted patients are summarized in

2 Supplementary Table 2.

3 At the time of analysis, 14 out of the 17 transplanted patients were alive and 13 remained disease-free after a median follow-up of 34.0 months from nivolumab initiation and 29.2 months from transplantation (Figure 3). The PFS and OS at one year from alloHSCT were $76 \%$ and $82 \%$, respectively.

In order to assess the benefit of alloHSCT consolidation, we compared the outcome of patients who achieved an objective response (CR or PR) upon nivolumab monotherapy and underwent $(\mathrm{N}=13)$ or not $(\mathrm{N}=37)$ immediate subsequent alloHSCT. Characteristics of these patients are summarized in Table 2. At nivolumab initiation, patients with subsequent alloHSCT were younger (median age $=30.5$ vs 38.0 years, $p=0.029$ ). There was no significant difference between the two groups regarding disease stage or number of prior lines of systemic therapy. The duration of treatment with nivolumab was shorter in patients receiving subsequent alloHSCT (median duration $=2.89$ vs 6.1 months, $p=0.012$ ) probably due to the initiation of the allograft procedure. The median time to response from nivolumab initiation was not significantly different between the two groups ( 2.0 vs 1.8 months, $p=0.420$ ) but the time to best response was shorter in the transplanted group (2.4 vs 1.8 months, $\mathrm{p}=0.039$ ). Indeed, all patients who underwent alloHSCT, except one, achieved a CR at first evaluation. At the time of analysis, 23 out of 37 (62.2\%) patients without subsequent alloHSCT had relapsed (7 out of $20 \mathrm{CR}$ patients and 16 out of 17 PR patients) whereas all patients who underwent alloHSCT remained disease-free after a median follow-up of 33.3 months from nivolumab initiation $(p<0.001)$. Median PFS was $22.4(95 \% \mathrm{Cl}[7.3 ; 32.0])$ months for the non-transplanted group and was not reached for the transplanted group $(p=0.003$; Figure $2 A)$. In terms of PFS, alloHSCT consolidation tended to benefit both $C R$ $(p=N S)$ and PR $(p=0.01)$ patients (Supplementary Figure 2 A \& C). In the non-transplanted group, 12 out of 20 (60\%) patients in CR and 0 out of 17 patients in PR were alive and progression-free at the time of analysis. In the transplanted group, 2 patients died of toxicity 
1 at day 155 and 192 after alloHSCT, respectively (causes of death are described below).

2 There was no difference in OS between the transplanted and non-transplanted groups

3 (Figure 2B). In the non-transplanted group, 19 out of 23 relapsed patients received a salvage

4 therapy. Among 12 patients evaluated, 9 (75\%) experienced an objective response including $5 \quad 5 \mathrm{CR}(41.7 \%)$.

All the 17 transplanted patients experienced acute and/or chronic-GVHD (Supplementary Table 2). Fourteen patients experienced acute GVHD (4 grade IV), 5 of which were steroidrefractory and required second line treatment with anti-IL2R antibody (inolimomab and basiliximab), anti-thymocyte globulin, or extra-corporal photopheresis. Seven patients experienced chronic GHVD, 5 of which were steroid-refractory and had not resolved at the time of analysis ( 4 cutaneous and 1 hepatic GVHD). One patient experienced veno-occlusive disease which resolved after defibrotide treatment and 2 patients presented a non-infectious febrile syndrome responding to corticosteroids. Treatment-Related Mortality (TRM) was $5.9 \%, 11.8 \%$ and $17.6 \%$ at day 100,6 months and 12 months, respectively. Three patients died: one patient had undergone prior alloHSCT and received a second (haploidentical) graft after reaching a PR upon nivolumab. He presented with a grade IV cutaneous and hepatic GHVD, and an unexplained encephalitis leading to death 5 months after transplantation. The second death occurred in a patient who underwent geno-identical alloHSCT after reaching a CR upon nivolumab. He first presented a non-infectious febrile syndrome that was efficiently treated with corticosteroids and then developed a steroid-refractory cutaneous and gastrointestinal GVHD. He died of massive and unexplained hemoptysis 6 months after transplantation. The third death occurred in a patient with infectious acute pneumonia.

\section{Discussion}

Our study has a particularly long follow-up compared to previously published studies (median $=34.3$ months versus $7-18$ months $[10,12,13])$. This gave us the opportunity to evaluate long 
1 term efficacy of anti-PD1 therapy in $78 \mathrm{R} / \mathrm{R} \mathrm{HL}$ patients. Although initial response to anti-PD1

2 was high $(\mathrm{ORR}=65.8 \%)$, our results show that most patients eventually relapse/progress, notably those who are unable to achieve a CR $(76 \%$ of all PR patients and $94 \%$ of PR patients without subsequent alloHSCT). In responding (CR or PR) patients, the relapse rate was significantly lower in patients consolidated with alloHSCT $(0 \%$ vs $62.2 \%, p<0.001)$. Furthermore, five out of 6 patients who were not in CR at the time of transplantation (4 PR and 1 PD) converted into a CR after alloHSCT.

Our results are consistent with the studies previously published by Armand et a[10], Chen et a[12] and Beköz et a[13] showing an ORR of $69 \%, 69 \%$, and 64\%, respectively (Supplementary Table 3). Similarly, the median PFS in our study was comparable to the one in the study by Armand et al (12.1 months versus 14.7 months, respectively).

We were also able to analyze the outcome of patients who underwent consolidation with alloHSCT and compare them to patients who were not consolidated with alloHSCT. To reduce the bias, we limited the final comparison to patients who experienced an objective response after nivolumab monotherapy.

In our cohort, 17 patients had undergone alloHSCT after nivolumab therapy (Figure 3). Among them, 13 patients were transplanted after reaching an objective response upon nivolumab monotherapy (9 CR and $4 \mathrm{PR}$ ). Of note, 4 additional patients were transplanted: one with a progressive disease and 3 after salvage therapy. Interestingly, 5 out of 6 patients who were not in CR at the time of transplantation (4 PR and $1 \mathrm{PD}$ ) converted into a CR after alloHSCT. Among the 17 transplanted patients, 14 are alive and 13 remain disease-free after a median follow-up of 29.2 months from transplantation. One-year PFS and OS from alloHSCT were $76 \%$ and $82 \%$, respectively. All patients experienced graft-versus-host disease (GVHD), either acute $(\mathrm{N}=14,82 \%)$ and/or chronic $(\mathrm{N}=7,41 \%)$ GVHD, including 7 (41\%) patients with grade III-IV GVHD. At the time of analysis, GVHD had resolved in 9 out of 13 disease-free patients. Two patients experienced non-infectious febrile syndrome which 
1 resolved with corticosteroids and one patient experienced a sinusoidal obstructive syndrome.

2 The 6 or 12 months TRM was $11.8 \%$ and $17.6 \%$.

3 Although the number of transplanted patients is limited, our results are consistent with other previously published studies (Supplementary Table 4). In their study, Merryman et al reported the outcome of 39 patients $(31 \mathrm{HL}$ and $8 \mathrm{NHL})$ who underwent alloHSCT after PD1 blockade[14]. Among HL patients, one-year PFS and OS were $74 \%$ and $90 \%$, respectively. Incidence of grade III-IV GVHD was $23 \%$ and 4 patients died of treatment-related toxicity. Seven patients (18\%) presented a non-infectious febrile syndrome requiring corticosteroids and 3 patients (8\%) a sinusoidal obstructive syndrome. In Checkmate-205, 44 patients out of 243 patients proceeded to alloHSCT, with a median follow-up of 5.5 months after alloHSCT[10]. The 6-months cumulative incidence of TRM and disease-progression were $13 \%$ and $7 \%$, respectively. All grade incidence of acute and chronic GVHD were $48 \%$ and $15 \%$, respectively.

To assess whether responding patients should be consolidated with alloHSCT or continue treatment with anti-PD1, we compared the outcome of patients in CR or PR upon nivolumab monotherapy with $(n=13)$ and without $(N=37)$ subsequent alloHSCT. Although the patients in the transplanted group were younger (median age $=30.5$ vs 38.0 years, $p=0.029$ ), and with a trend for more CR, the other characteristics at baseline were not significantly different between the 2 groups (Table 2). Twelve out of 20 patients (60\%) who achieved a CR upon nivolumab monotherapy and did not undergo alloHSCT remained disease-free at the time of analysis, suggesting that some of them may be cured with anti-PD1 alone, even after nivolumab discontinuation[11]. Conversely, 16 out of $17(94.1 \%)$ patients who were unable to reach a CR upon nivolumab and did not undergo alloHSCT eventually progressed (Supplementary Figure 2). In transplanted patients, the relapse rate was markedly lower compared to non-transplanted patients ( $0 \%$ vs $62.2 \%, p<0.001$, Figure $2 A)$, both in $C R$ and PR patients (Supplementary Figure 2). Interestingly, 5 out of 6 patients who were not in CR at the time of transplantation (4 PR and $1 \mathrm{PD}$ ) converted into a CR after alloHSCT. There 
was no significant difference in OS between the transplanted and non-transplanted groups (Figure 2B). Whether the benefit observed in PFS may eventually translate into an increase in OS remains uncertain and should be re-evaluated after a longer follow-up. Emerging data suggest that anti-PD1 therapy may "re-sensitize" Hodgkin lymphoma tumors to standard chemotherapy[17]. Thus, patients who relapse or progress after anti-PD1 therapy may respond to salvage chemotherapy even if they were previously refractory. In our cohort, despite a great number of prior lines of treatment, most patients who progressed/relapsed after anti-PD1 were able to receive a salvage therapy (80\%), most of whom achieved an objective response (56.5\% among evaluated patients). These results suggest that a subset of patients who fail anti-PD1 therapy may still be rescued.

The relapse rate after alloHSCT following anti-PD1 therapy seems much lower compared to patients who are transplanted after conventional chemotherapy. Indeed, the one-year relapse rate in our study was $5.8 \%$ versus $26-41 \%$ in historical controls[18-24], suggesting that prior anti-PD1 therapy may enhance the graft-versus-lymphoma effect of alloHSCT.

Although our study suggests a benefit of alloHSCT consolidation, these results should be interpreted with caution given the retrospective nature of this analysis. Despite our attempt to limit the bias associated with the response to anti-PD1 therapy by limiting the comparison to responding patients, other biases - including age and proportion of complete responders may have influenced the differences observed between transplanted and non-transplanted groups. The decision to perform an alloHSCT was left to the decision of the primary physician and thus could not be controlled. Finally, the number of patients in the two subgroups is limited and may have reduced the power of the study.

Overall, our results show that, with a long follow-up (median $=34.3$ months), most R/R HL patients eventually progress during anti-PD1 therapy, notably patients who are unable to achieve a CR. Conversely, consolidation with alloHSCT can convert incomplete responses into $\mathrm{CR}$ and is associated with a very low relapse-rate. Although patients undergoing alloHSCT after anti-PD1 may experience increased toxicities, these adverse events 
1 appeared manageable and reversible in most patients. Thus, consolidation with alloHSCT

2 may be considered in R/R HL treated with anti-PD1, particularly in those not reaching a CR.

3 However, the absence of benefit in OS and the possibility to induce a response with salvage

4 chemotherapy after anti-PD1 "re-sensitization" should be considered when evaluating the

5 risk/benefit ratio. Prospective studies are needed to further define which patients may benefit

6 from consolidation with alloHSCT after anti-PD1 therapy.

7 


\section{Acknowledgments}

Elodie Gat, Laure Flament, Fanny Cherblanc, Florence Broussais, Nadine Morineau, Jean-

Pierre Marolleau, Elena Loppinet, Sophie Lefort, Isabelle Roche-Lachaise, Sophie

Ducastelle, Benoît Bareau, Mohamed Touati, Franck Morschhauser, Fabien Lebras, Marjan

Ertault, Frédéric Peyrade, Caroline Regny, Thomas Gastine, Julien Lazarovici, Stéphanie

Guidez, Luc-Mathieu Fornecker, Katell Le Dû, Georges Garnier, Barbara Burroni, Aiping Chen and Fatima-Zohra Mokrane.

\section{Authorship contributions}

G.M. and R.H. designed the research, analyzed data, and wrote the paper; C.H., JM.S, O.C., A.St., B.D., A.Sh., G.G., L.M., K.B., MP.M.-M., H.G., A.T., R.D., E.N.-V., A.D., C.B., A.C., P.B., provided the data; and all authors reviewed and approved the final draft.

\section{Funding}

This study was supported and funded by Bristol-Myers Squibb. The views expressed in this article are the authors' own and not an official position of Bristol-Myers Squibb or their respective institutions.

\section{Conflict of interest disclosures}

R.H. and P.B. have received consulting fees and/or honoraria from Bristol-Myers Squibb. E.N-V has received consulting fees from Keocyt, Janssen and Sanofi, A.St. has received consulting fees from Takeda. The remaining authors declare no competing financial interests. 


\section{References}

[1] Gallamini A, Tarella C, Viviani S, Rossi A, Patti C, Mule A, et al. Early chemotherapy intensification with escalated beacopp in patients with advanced-stage hodgkin lymphoma with a positive interim positron emission tomography/computed tomography scan after two abvd cycles: Long-term results of the GITIL/FIL HD 0607 tria. J Clin Oncol 2018;36:454-62. doi:10.1200/JCO.2017.75.2543.

[2] Press OW, Li H, Schöder H, Straus DJ, Moskowitz $\mathrm{CH}$, LeBlanc $\mathrm{M}$, et al. US intergroup trial of response-adapted therapy for stage III to IV Hodgkin lymphoma using early interim fluorodeoxyglucose-positron emission tomography imaging: Southwest Oncology Group S0816. J Clin Oncol 2016;34:2020-7. doi:10.1200/JCO.2015.63.1119.

[3] Rancea M, Monsef I, von Tresckow B, Engert A, Skoetz N. High-dose chemotherapy followed by autologous stem cell transplantation for patients with relapsed/refractory Hodgkin lymphoma. Cochrane Database Syst Rev 2013:CD009411. doi:10.1002/14651858.CD009411.pub2.

[4] Arai S, Fanale M, DeVos S, Engert A, Illidge T, Borchmann P, et al. Defining a Hodgkin lymphoma population for novel therapeutics after relapse from autologous hematopoietic cell transplant. Leuk Lymphoma 2013;54:2531-3. doi:10.3109/10428194.2013.798868.

[5] Crump M. Management of Hodgkin lymphoma in relapse after autologous stem cell transplant. Hematol Am Soc Hematol Educ Progr 2008;2008:326-33. doi:10.1182/asheducation-2008.1.326.

[6] Chen R, Gopal AK, Smith SE, Ansell SM, Rosenblatt JD, Savage KJ, et al. Five-year survival and durability results of brentuximab vedotin in patients with relapsed or refractory Hodgkin lymphoma. Blood 2016;128:1562-6. doi:10.1182/blood-2016-02699850.

[7] Ansell SM, Lesokhin AM, Borrello I, Halwani A, Scott EC, Gutierrez M, et al. PD-1 Blockade with Nivolumab in Relapsed or Refractory Hodgkin's Lymphoma. N Engl J Med 2015;372:311-9. doi:10.1056/NEJMoa1411087.

[8] Armand P, Shipp MA, Ribrag V, Michot J-M, Zinzani PL, Kuruvilla J, et al. Programmed Death-1 Blockade With Pembrolizumab in Patients With Classical Hodgkin Lymphoma After Brentuximab Vedotin Failure. J Clin Oncol 2016;34:3733-9. doi:10.1200/JCO.2016.67.3467.

[9] Engert A, Fanale M, Santoro A, Armand P, Ansell SM. Nivolumab for relapsed/refractory classical Hodgkin lymphoma after autologous transplant: full results after extended follow-up of the multicohort muticenter phase 2 CHECKMATE 205 trial. Proceedings of the 22th annual congress of the EHA. Absract S412, Madrid, Spain: 2017.

[10] Armand P, Engert A, Younes A, Fanale M, Santoro A, Zinzani PL, et al. Nivolumab for Relapsed/Refractory Classic Hodgkin Lymphoma After Failure of Autologous Hematopoietic Cell Transplantation: Extended Follow-Up of the Multicohort Single-Arm Phase II CheckMate 205 Trial. J Clin Oncol 2018:JCO.2017.76.079. doi:10.1200/JCO.2017.76.0793.

[11] Manson G, Herbaux C, Brice P, Bouabdallah K, Stamatoullas A, Schiano J-M, et al. Prolonged remissions after anti-PD1 discontinuation in patients with Hodgkin lymphoma. Blood 2018;33:blood-2018-03-841262. doi:10.1182/blood-2018-03841262. 
[12] Chen R, Zinzani PL, Fanale MA, Armand P, Johnson NA, Brice P, et al. Phase II Study of the Efficacy and Safety of Pembrolizumab for Relapsed/Refractory Classic Hodgkin Lymphoma. J Clin Oncol 2017;35:2125-32. doi:10.1200/JCO.2016.72.1316.

[13] Beköz H, Karadurmuş N, Paydaş S, Türker A, Toptaş T, Fıratlı Tuğlular T, et al. Nivolumab for relapsed or refractory Hodgkin lymphoma: real-life experience. Ann Oncol 2017;28:2496-502. doi:10.1093/annonc/mdx341.

[14] Merryman RW, Kim HT, Zinzani PL, Carlo-Stella C, Ansell SM, Perales M-A, et al. Safety and efficacy of allogeneic hematopoietic stem cell transplant after PD-1 blockade in relapsed/refractory lymphoma. Blood 2017;129:1380-8. doi:10.1182/blood-2016-09-738385.

[15] Cheson BD, Horning SJ, Coiffier B, Shipp MA, Fisher RI, Connors JM, et al. Report of an international workshop to standardize response criteria for non-Hodgkin's lymphomas. J Clin Oncol 1999;17:1244-53. doi:10.1200/JCO.1999.17.4.1244.

[16] Cheson BD, Pfistner B, Juweid ME, Gascoyne RD, Specht L, Horning SJ, et al. Revised response criteria for malignant lymphoma. J Clin Oncol 2007;25:579-86. doi:10.1200/JCO.2006.09.2403.

[17] Rossi C, Gilhodes J, Maerevoet M, Herbaux C, Morschhauser F, Brice P, et al. Efficacy of chemotherapy or chemo-anti-PD-1 combination after failed anti-PD-1 therapy for relapsed and refractory hodgkin lymphoma: A series from lysa centers. Am J Hematol 2018;93:1042-9. doi:10.1002/ajh.25154.

[18] Anderlini P, Saliba RM, Ledesma C, Plair T, Alousi AM, Hosing CM, et al. Gemcitabine, Fludarabine, and Melphalan for Reduced-Intensity Conditioning and Allogeneic Stem Cell Transplantation for Relapsed and Refractory Hodgkin Lymphoma. Biol Blood Marrow Transplant 2016;22:1333-7. doi:10.1016/j.bbmt.2016.03.028.

[19] Devetten MP, Hari PN, Carreras J, Logan BR, van Besien K, Bredeson CN, et al. Unrelated Donor Reduced-Intensity Allogeneic Hematopoietic Stem Cell Transplantation for Relapsed and Refractory Hodgkin Lymphoma. Biol Blood Marrow Transplant 2009;15:109-17. doi:10.1016/j.bbmt.2008.11.011.

[20] Marcais A, Porcher R, Robin M, Mohty M, Michalet M, Blaise D, et al. Impact of disease status and stem cell source on the results of reduced intensity conditioning transplant for Hodgkin's lymphoma: A retrospective study from the French Society of Bone Marrow Transplantation and Cellular Therapy (SFGM-TC). Haematologica 2013;98:1467-75. doi:10.3324/haematol.2012.080895.

[21] Sureda A, Robinson S, Canals C, Carella AM, Boogaerts MA, Caballero D, et al. Reduced-intensity conditioning compared with conventional allogeneic stem-cell transplantation in relapsed or refractory Hodgkin's lymphoma: An analysis from the lymphoma working party of the European Group for Blood and Marrow Transplantation. J Clin Oncol 2008;26:455-62. doi:10.1200/JCO.2007.13.2415.

[22] Armand P, Kim HT, Sainvil MM, Lange PB, Giardino AA, Bachanova V, et al. The addition of sirolimus to the graft-versus-host disease prophylaxis regimen in reduced intensity allogeneic stem cell transplantation for lymphoma: A multicentre randomized trial. Br J Haematol 2016;173:96-104. doi:10.1111/bjh.13931.

[23] Armand P, Kim HT, Ho VT, Cutler CS, Koreth J, Antin JH, et al. Allogeneic Transplantation with Reduced-Intensity Conditioning for Hodgkin and non-Hodgkin Lymphoma: Importance of Histology for Outcome. Biol Blood Marrow Transplant 2008;14:418-25. doi:10.1016/j.bbmt.2008.01.008. 
[24] Robinson SP, Sureda A, Canals C, Russell N, Caballero D, Bacigalupo A, et al. Reduced intensity conditioning allogeneic stem cell transplantation for Hodgkin's lymphoma: identification of prognostic factors predicting outcome. Haematologica 2009;94:230-8. doi:10.3324/haematol.13441. 
Figure 1: PFS (A \& $C)$ and $O S(B \& D)$ of the entire cohort $(A \& B)$ and according to best response upon nivolumab monotherapy $(C \& D)$

Figure 2: PFS (A) and OS (B) of patients in CR or PR after nivolumab monotherapy according to subsequent allogenic-HSCT

Figure 3: Outcome of patients who underwent allogenic HSCT after nivolumab therapy 
Table 1. Patients' characteristics for the entire cohort

\begin{tabular}{|c|c|}
\hline Characteristics at nivolumab initiation & $\begin{array}{l}\text { All } \\
N=78\end{array}$ \\
\hline Age, years, median [range] & $37.0[18-77]$ \\
\hline $\begin{array}{c}\text { Sex, No }(\%) \\
\qquad \quad \text { Male } \\
\cdot \quad \text { Female }\end{array}$ & $\begin{array}{l}44(56.4 \%) \\
34(43.6 \%)\end{array}$ \\
\hline $\begin{array}{cl}\text { Performance status (ECOG) } \\
\text { - } \quad 0-1 \\
-\quad \geq 2 \\
\cdot \quad \text { Missing }\end{array}$ & $\begin{array}{l}45(72.6 \%) \\
17(27.4 \%) \\
16\end{array}$ \\
\hline $\begin{array}{cl}\text { Stage disease, No (\%) } \\
\qquad \quad \mathrm{I} / \mathrm{II} \\
\bullet \quad \mathrm{II} / \mathrm{IV} \\
\bullet \quad \text { Unknown }\end{array}$ & $\begin{array}{l}16(22.5 \%) \\
55(77.5 \%) \\
7\end{array}$ \\
\hline 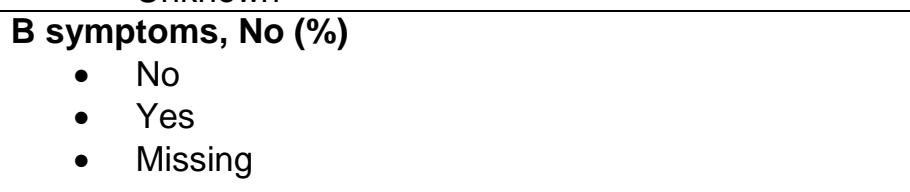 & $\begin{array}{l}54(73 \%) \\
20(27 \%) \\
4\end{array}$ \\
\hline 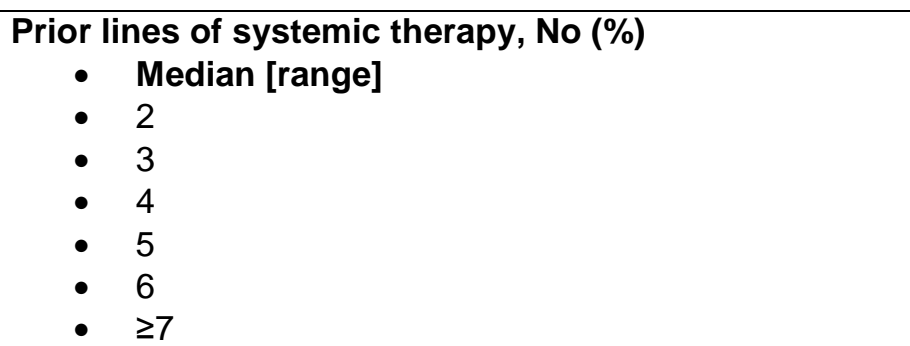 & $\begin{array}{l}6[2-13] \\
2(2.6 \%) \\
9(11.5 \%) \\
11(14.1 \%) \\
11(14.1 \%) \\
13(16.7 \%) \\
32(41.0 \%)\end{array}$ \\
\hline Prior radiation therapy, No (\%) & $42(53.8 \%)$ \\
\hline Prior treatment with Brentuximab Vedotin, No (\%) & $\begin{array}{l}74(100 \%) \\
4 \text { missing }\end{array}$ \\
\hline Prior autologous HSCT, No (\%) & $48(61.5 \%)$ \\
\hline Prior allogenic HSCT, No (\%) & $22(28.2 \%)$ \\
\hline Nivolumab treatment and response & \\
\hline $\begin{array}{l}\text { Number of nivolumab injections, median [range] } \\
\text { Duration of anti-PD1 therapy, months, median [range] }\end{array}$ & $\frac{9.5[1-84]}{5.2[0-38.3]}$ \\
\hline Permanent treatment discontinuation & $67(85.9 \%)$ \\
\hline $\begin{array}{l}\text { Reason for treatment discontinuation } \\
\text { - } \quad \text { Disease progression } \\
\text { - } \quad \text { Toxicity } \\
\text { - } \quad \text { Consolidation with allogenic-HSCT } \\
\text { - } \quad \text { Consolidation with autologous transplant } \\
\text { - } \quad \text { Decision of the clinician with no further treatment } \\
\text { - } \quad \text { Other }\end{array}$ & $\begin{array}{l}34(49.3 \%) \\
6(9.0 \%) \\
13(20.9 \%) \\
1(1.5 \%) \\
7(10.4 \%) \\
2(3.0 \%) \\
4(6.0 \%)\end{array}$ \\
\hline Concomitant radiotherapy, No (\%) & $7(9.6 \%)$ \\
\hline Concomitant chemotherapy, No (\%) & $5(6.8 \%)$ \\
\hline Follow-up, months, median [range] & $34.3[0.1-39.7]$ \\
\hline
\end{tabular}




\begin{tabular}{|c|c|}
\hline $\begin{aligned} \text { BOR among evaluated patients, No (\%) } \\
-\quad \text { CR } \\
-\quad \text { PR } \\
-\quad \text { SD } \\
-\quad \text { PD } \\
-\quad \text { Non-evaluated/missing }\end{aligned}$ & $\begin{array}{l}29(38.2 \%) \\
21(27.6 \%) \\
11(14.5 \%) \\
15(19.7 \%) \\
2\end{array}$ \\
\hline Time to response from Nivo initiation, median [range] & $2.6[0.5-34.1]$ \\
\hline $\begin{array}{l}\text { Time to best response from Nivo initiation, median } \\
\text { [range] }\end{array}$ & $2.7[0.5-28.8]$ \\
\hline DOR, median $(95 \% \mathrm{Cl})$ & $24.3(9.9-\mathrm{NE})$ \\
\hline PFS, median (95\% Cl) & $12.1(7.3-26.2)$ \\
\hline OS, median (95\% Cl) & $38.7(38.7-\mathrm{NE})$ \\
\hline Relapse / progression & $45(57.7 \%)$ \\
\hline If relapse/progression, salvage therapy & $36(80 \%)$ \\
\hline $\begin{array}{l}\text { Response after salvage therapy } \\
\text { CR } \\
\text { PR } \\
\text { SD } \\
\text { PD } \\
\text { Non-evaluated/missing }\end{array}$ & $\begin{array}{l}8(34.8 \%) \\
5(21.7 \%) \\
1(4.3 \%) \\
9(39.1 \%) \\
13\end{array}$ \\
\hline
\end{tabular}

NE, Not evaluable 


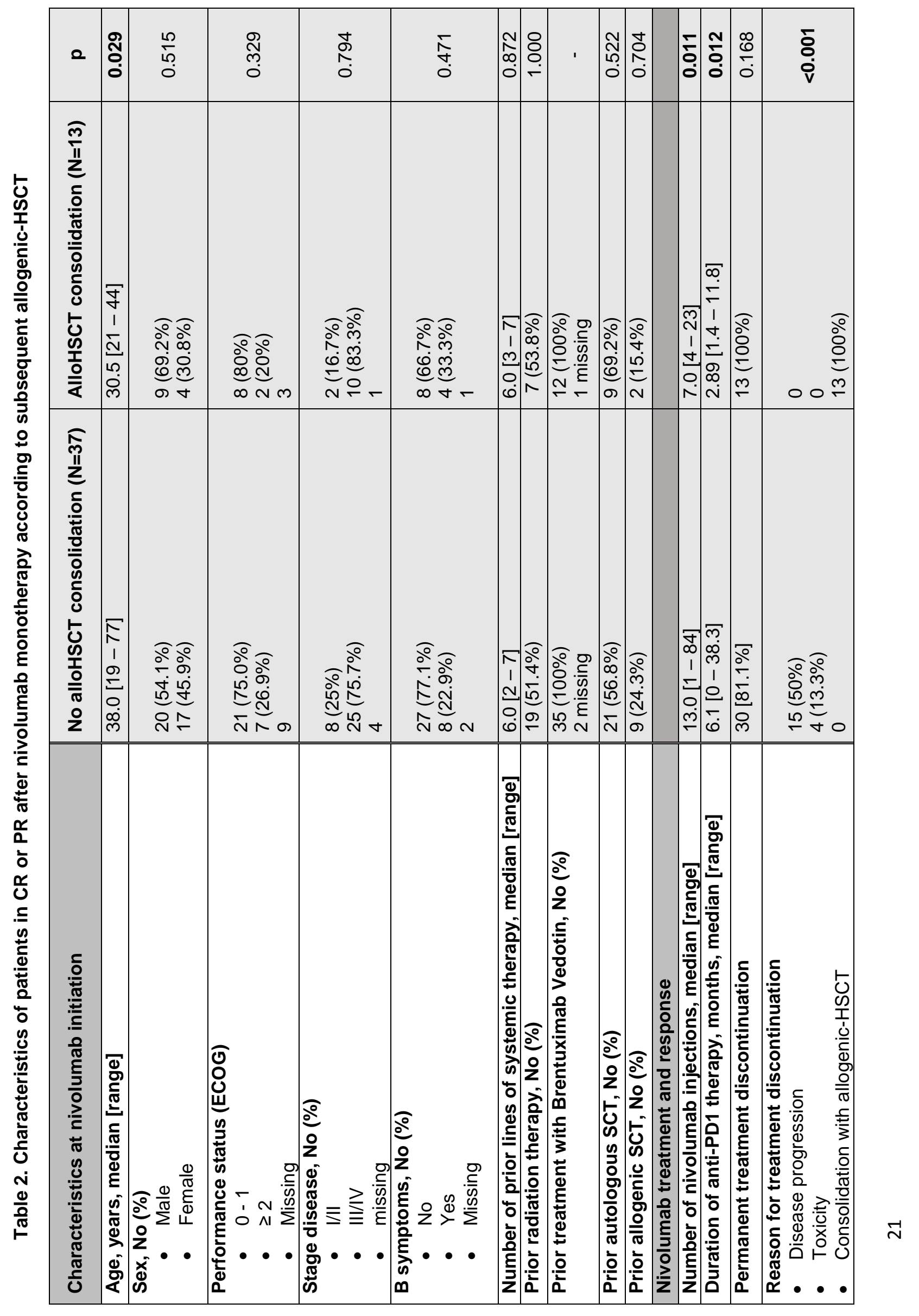




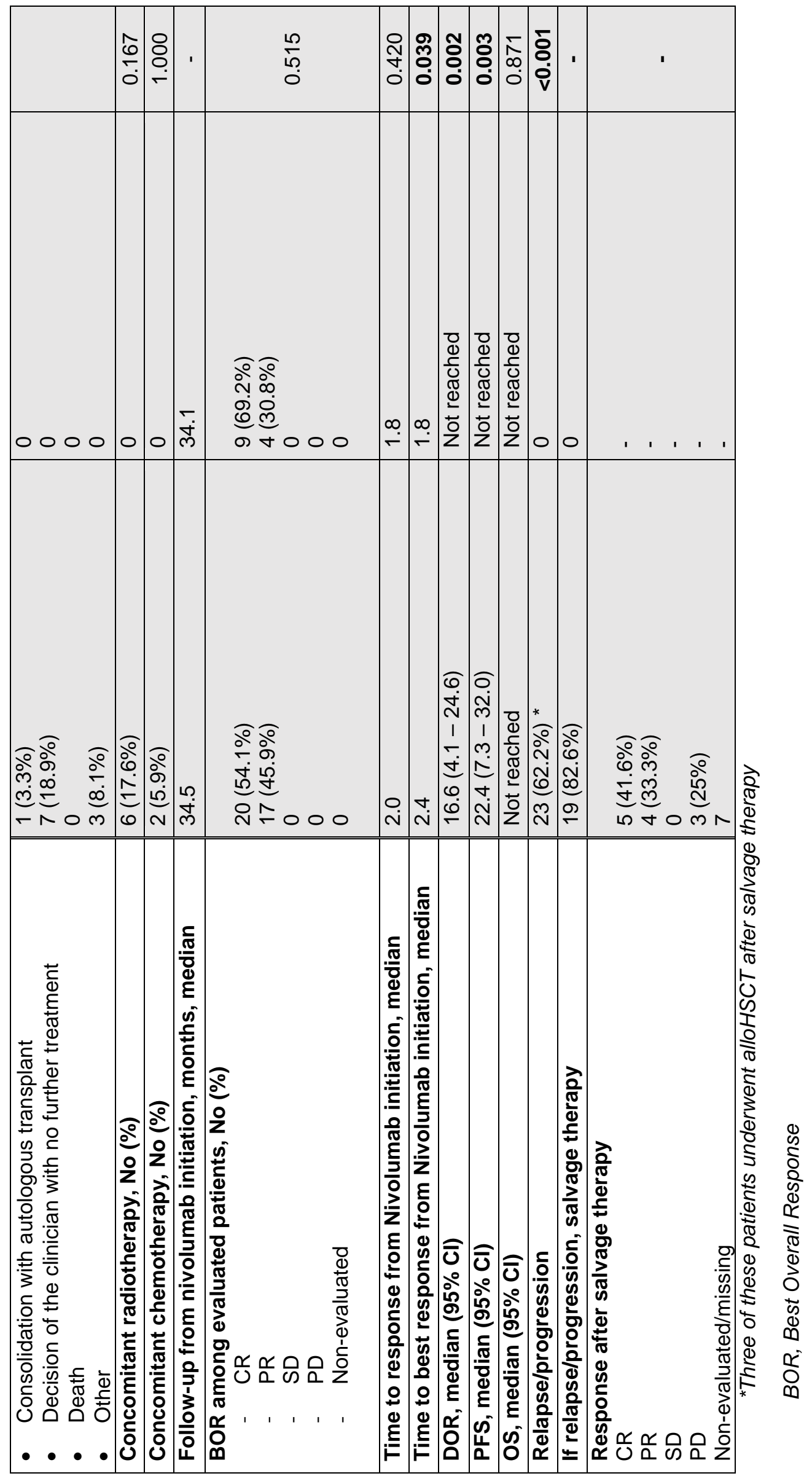




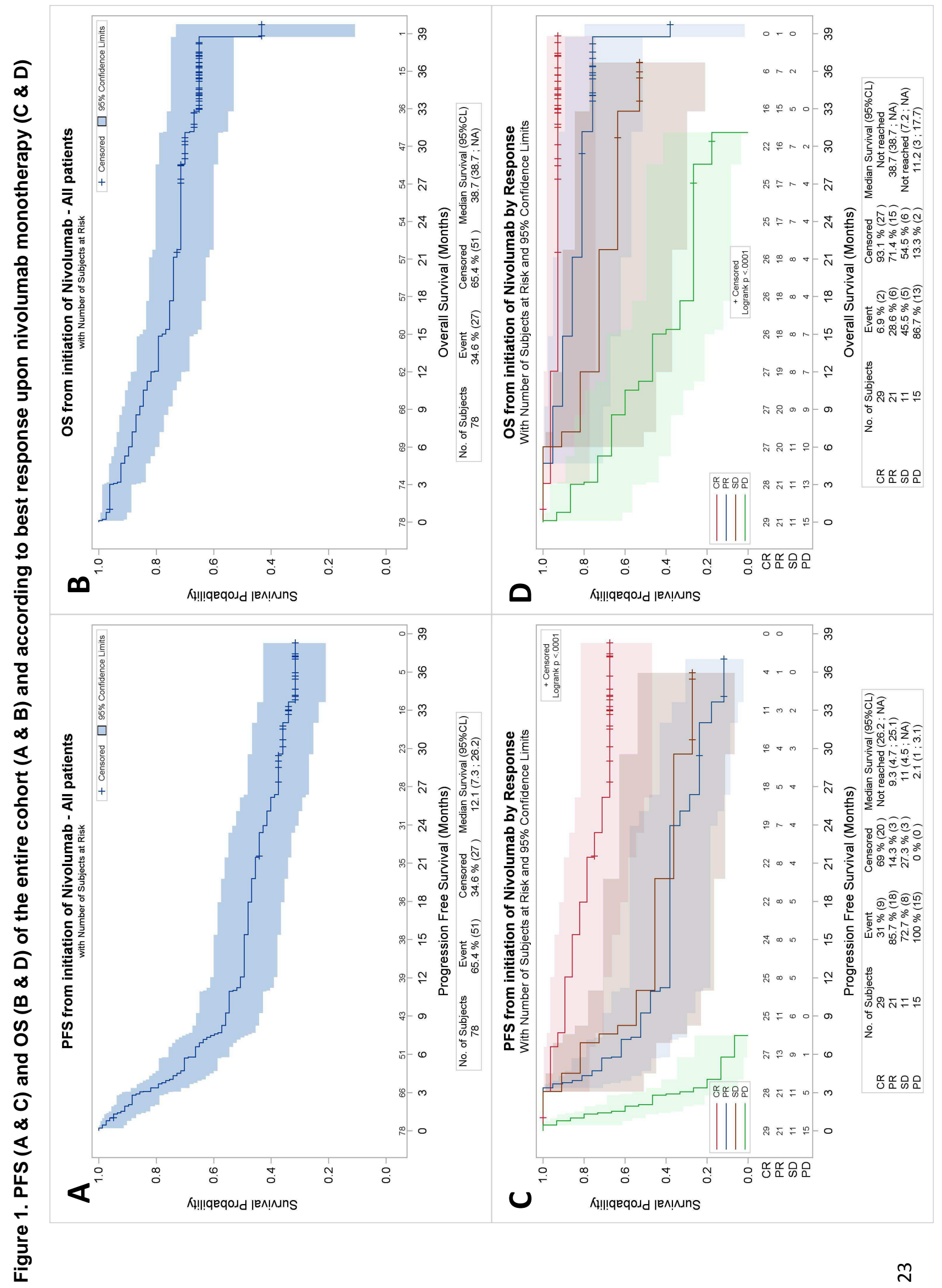




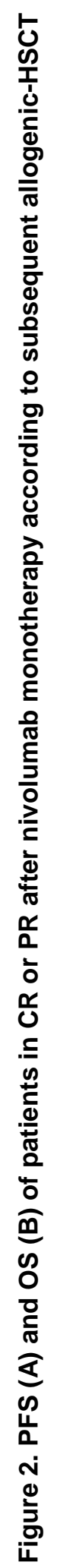
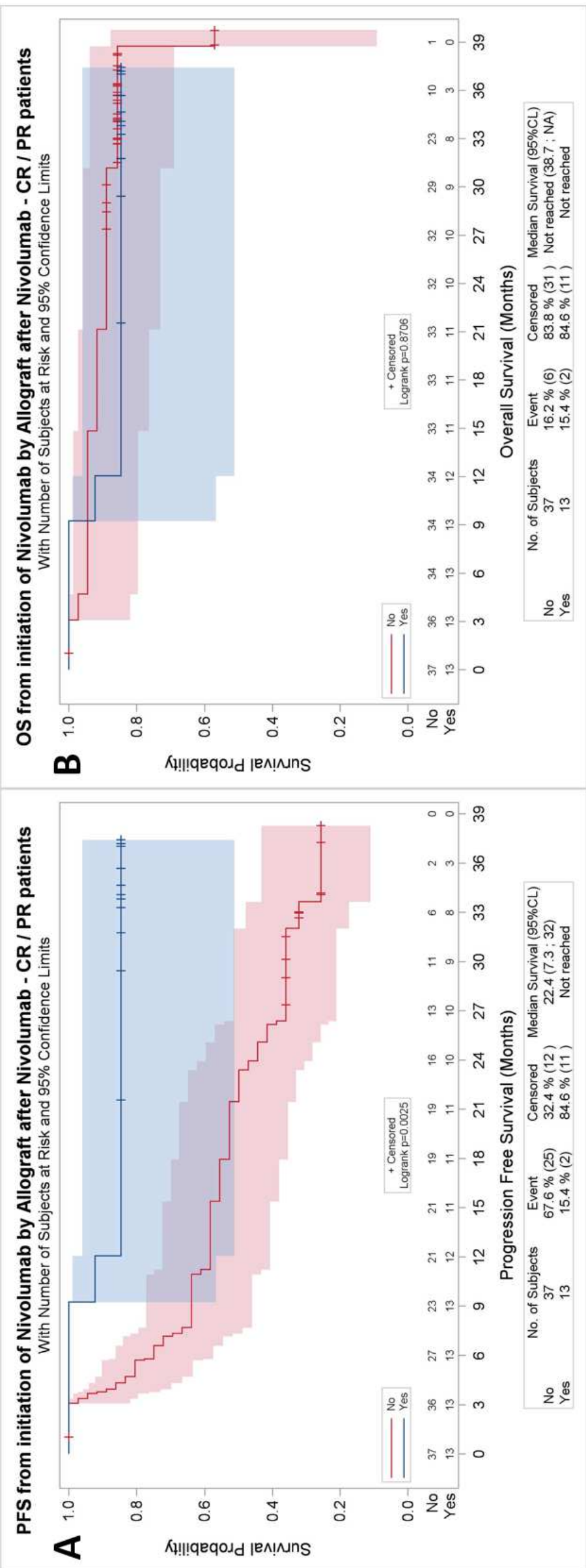


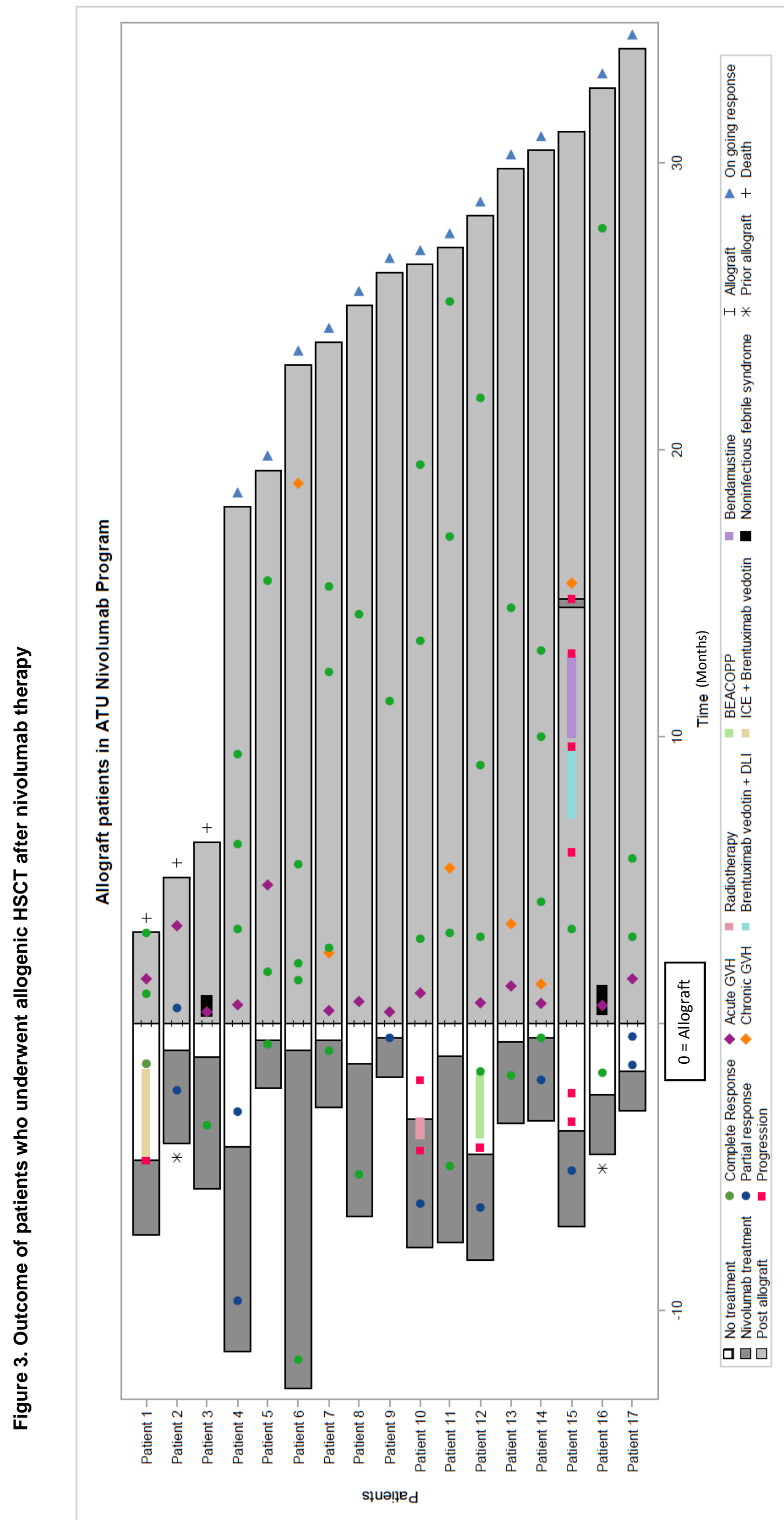

